\title{
Esophageal cancer: comparative effectiveness of treatment options
}

\author{
This article was published in the following Dove Press journal: \\ Comparative Effectiveness Research \\ 13 April 2016 \\ Number of times this article has been viewed
}

\author{
Cai $\mathrm{Xu}{ }^{\prime}$ \\ Steven H Lin² \\ 'Department of Radiation Oncology, \\ Cancer Hospital and Institute, \\ Chinese Academy of Medical Sciences \\ Peking Union Medical College, \\ Beijing, People's Republic of China; \\ ${ }^{2}$ Department of Radiation Oncology, \\ The University of Texas MD Anderson \\ Cancer Center, Houston, TX, USA
}

Correspondence: Steven H Lin

Department of Radiation Oncology,

The University of Texas MD Anderson

Cancer Center, 1515 Holcombe Blvd.,

Unit 097, Houston, Texas 77030, USA

Tel +l 7I35638490

Fax + I 7|35632366

Emailshlin@mdanderson.org

\begin{abstract}
Esophageal cancer is a lethal disease. Multimodal therapy has improved the survival and local control for locally advanced esophageal cancer compared to surgery alone. Neoadjuvant chemoradiotherapy (CRT) plus surgery is the accepted standard of care for operable esophageal cancer. Pathologic complete response is very common, ranging from $18 \%$ to $43 \%$, and it is an independent favorable prognostic factor for survival. Salvage surgery after definitive CRT may be safe and feasible for patients with esophageal cancer who achieve pathologic complete response after neoadjuvant CRT. For inoperable cases, definitive CRT is an alternative treatment modality. Intensity-modulated radiation therapy may reduce treatment-related toxicities due to its better dose homogeneity and conformity compared to three-dimensional conformal radiotherapy. Proton therapy can further improve target conformity and normal tissue sparing when compared to photon therapy (three-dimensional conformal radiotherapy and intensitymodulated radiation therapy) and, therefore, likely to further improve the treatment outcomes of patients. This article compares the effectiveness of the various treatment approaches in the definitive or neoadjuvant setting and reviews the evidence on the benefit of utilizing advanced radiation techniques for the curative management of esophageal cancer.
\end{abstract}

Keywords: esophageal cancer, treatment modalities, comparative effectiveness, IMRT, proton therapy

\section{Introduction}

Esophageal cancer is the eighth most common cancer worldwide and the sixth most common cause of cancer death. ${ }^{1}$ It is a highly lethal disease with poor prognosis and imposes a high health burden in many societies. Esophageal cancer is mainly classified as squamous-cell carcinoma (SCC) and adenocarcinoma (AC). Traditionally, surgery alone is the standard treatment for localized SCC and AC of the esophagus, especially for patients with early-stage diseases. However, despite the remarkable improvement in surgical techniques, the survival with surgery alone in locally advanced esophageal cancer remains poor, with median 5-year overall survival (OS) of $\sim 20 \% .^{2-4}$ It has unacceptably high rates of locoregional recurrence (LRR), despite R0 resection, ranging from $41.5 \%$ to $49 \% .^{5-8}$ This has prompted the introduction of multimodal therapy to improve the results, such as definitive chemoradiotherapy (CRT), pre- and postoperative CRT, pre- and postoperative chemotherapy, and pre- and postoperative radiotherapy. Over the past 3 decades, surgical advances and improvement in radiation techniques have greatly improved the disease outcomes. ${ }^{9-11}$ This article focuses on integrating these approaches and tailoring the treatment strategy for individual patients, compares the effectiveness of the various treatment approaches in the definitive or neoadjuvant 
setting, and reviews the evidence on the benefit of utilizing advanced radiation techniques for the curative management of esophageal cancer.

\section{Definitive treatment for esophageal cancer}

Historically, surgery has been the primary treatment modality for esophageal cancer, especially for patients with early-stage disease. However, in patients with medical comorbid conditions or unresectable diseases, nonsurgery treatment modalities, such as radiotherapy, will be a cornerstone of therapy. Radiotherapy alone has historically been used as an alternative curative treatment approach for patients with these conditions. However, concurrent CRT (CCRT) has now become the standard nonsurgery treatment option for patients who could tolerate chemotherapy.

\section{Definitive radiotherapy alone vs definitive CRT for esophageal cancer}

The prognosis is very poor when radiotherapy alone is used. ${ }^{12}$ Cisplatin-based CCRT, as definitive treatment for unresectable esophageal cancer, has improved the survival compared to definitive radiotherapy alone, although at the cost of increased toxicity (Table 1 ). ${ }^{12-16}$ An early randomized trial showed that CCRT was superior to radiotherapy alone in patients with localized esophageal cancer, in terms of survival, local control, and distant metastases. ${ }^{14}$ The landmark intergroup study Radiation Therapy Oncology Group (RTOG) 85-01 demonstrated that CCRT significantly increased OS in patients with T1-3N0-1M0 SCC or AC of the esophagus compared to radiotherapy alone. ${ }^{12}$ However, acute toxicity was higher in the CCRT group, yet there were no differences in the long-term toxicity. ${ }^{12}$ Recently, a meta-analysis was conducted, which included nine randomized studies accounting for 1,135 patients (CCRT: 612; radiotherapy alone: 523) with $97.4 \%$ being SCC, and indicated that CCRT is the most effective approach for treating locally advanced esophageal cancer with the improved survival and local control rates compared to radiotherapy alone. ${ }^{17}$

\section{Surgery vs definitive radiotherapy alone for esophageal cancer}

Surgery is still considered the most effective single modality therapy for the management of esophageal cancer, while radiotherapy also plays an important role in the curative management of inoperable esophageal cancer. A few studies have demonstrated that surgery alone for esophageal cancer was superior to definitive radiotherapy. A large retrospective study from Taiwan reviewed outcomes from 12,482 patients with $>90 \%$ esophageal SCC and reported that surgery significantly improves survival compared to definitive radiotherapy. ${ }^{18}$ In a small randomized study of 99 patients with operable SCC of the esophagus, the results showed that surgery was superior to radiotherapy alone in improving the quality of swallowing $(P=0.03)$ and survival $(P=0.002) .{ }^{19}$ Although surgery is the preferred option over radiation alone, radiation therapy alone is primarily a palliative option for patients who are unable to tolerate chemotherapy.

\section{Surgery vs definitive CRT for esophageal cancer}

Phase III randomized trials comparing CCRT with radiation alone ${ }^{12-16}$ have made CCRT the standard treatment for patients with inoperable esophageal cancer. However, there are only a few studies comparing the definitive CCRT with surgery alone for esophageal cancer, most of which are prospective Phases I and II clinical trials and retrospective studies, ${ }^{20-22}$ and only a few of which are randomized clinical trials. ${ }^{23}$ Teoh et $\mathrm{al}^{23}$ reported a randomized trial of 81 patients with operable SCC of the mid- or lower thoracic esophagus with a 5-year OS favoring CRT, although it was not statistically significant (surgery $29.4 \%$ and CRT $50 \% ; P=0.147$ ). It was concluded that the long-term survival of definitive CRT for SCC was at least comparable to surgery. A meta-analysis showed that OS was equivalent between surgery and defini-

Table I Studies of definitive RT alone vs definitive CRT for esophageal cancer

\begin{tabular}{|c|c|c|c|c|c|c|c|c|c|c|}
\hline \multirow[t]{2}{*}{ References } & \multicolumn{2}{|l|}{$\mathbf{N}$} & \multicolumn{2}{|l|}{$\begin{array}{l}\text { RT dose } \\
\text { (Gy) }\end{array}$} & \multirow[t]{2}{*}{ Chemotherapy } & \multicolumn{2}{|c|}{$\begin{array}{l}\text { Median OS } \\
\text { (months) }\end{array}$} & \multicolumn{2}{|c|}{$\begin{array}{l}\text { 5-year OS } \\
\text { (\%) }\end{array}$} & \multirow[t]{2}{*}{$P$-value } \\
\hline & $\mathbf{R T}$ & CRT & RT & CRT & & RT & CRT & RT & CRT & \\
\hline Cooper et al ${ }^{12}$ & 62 & 61 & 64 & 50 & $\mathrm{DDP}+5-\mathrm{FU}$ & - & - & 0 & 26 & \\
\hline Al-Sarraf et al ${ }^{13}$ & 62 & 61 & 64 & 50 & $\mathrm{DDP}+5-\mathrm{FU}$ & 9.3 & 14.1 & 0 & 27 & $<0.0001$ \\
\hline Herskovic et al ${ }^{14}$ & 60 & 61 & 64 & 50 & $\mathrm{DDP}+5-\mathrm{FU}$ & 8.9 & 12.5 & - & - & $<0.001$ \\
\hline Smith et al ${ }^{15}$ & 60 & 59 & $40+S ; 60$ & $40+S ; 60$ & $\mathrm{MMC}+5-\mathrm{FU}$ & 9.2 & 14.8 & 7 & 9 & $<0.05$ \\
\hline Smit et $\mathrm{al}^{16}$ & 177 & 110 & 60 & 50.4 & DDP based & - & - & - & - & 0.071 \\
\hline
\end{tabular}

Abbreviations: CRT, chemoradiotherapy; DDP, cisplatin; 5-FU, fluorouracil; OS, overall survival; RT, radiotherapy; S, surgery; MMC, mitomycin. 
tive CRT $(P=0.84) .{ }^{24}$ The similar outcome in survival between surgery alone and CRT suggests that a nonsurgical approach of definitive CRT is a reasonable choice, especially in patients with esophageal cancer who have inoperable diseases or refuse surgery.

\section{Multimodal therapy for esophageal cancer \\ Neoadjuvant CRT plus surgery vs surgery alone for esophageal cancer}

Neoadjuvant CRT has been extensively studied in the prospective trials. The evidence to date has suggested that this approach should be integrated into the standard treatment of patients with locally advanced and operable esophageal cancer. A total of 12 randomized trials investigating preoperative CRT compared to surgery alone were identified (Table 2). ${ }^{11,25-36}$ Among the 12 randomized trials, preoperative CRT improves the OS rate of patients with esophageal cancer in only five trials. ${ }^{11,29,30,33,35}$ Despite some studies not showing the survival benefit, the advantages of preoperative CRT could be illustrated in certain aspects, such as improved local control rate ${ }^{25,29}$ and better complete resection rate. ${ }^{25,28,30}$ The Irish trial ${ }^{33}$ was a small randomized trial in 113 patients with $\mathrm{AC}$ randomized to surgery alone vs preoperative CRT with two cycles of cisplatin/fluorouracil and 40 Gy in 15 fractions. Although the study demonstrated an improved survival with preoperative CRT, the uncharacteristic poor survival in the surgery alone group (6\% vs $32 \%$ at 3 years) confounded the benefit of adding CRT. The Cancer and Leukemia Group B (CALGB) 9781 study closed early after only 56 patients were enrolled but reported an improved OS with a median survival of 4.48 years vs 1.79 years $(P=0.002)$ and 5 -year survival of $39 \%$ vs $16 \%$, in favor of preoperative CRT. ${ }^{11}$ The Chemoradiotherapy for Oesophageal Cancer Followed by Surgery Study (CROSS) that compared neoadjuvant CRT vs surgery alone in patients with $\mathrm{SCC}$ and $\mathrm{AC}$ of the esophagus or esophagogastric junction (EGJ) showed a significant improved OS and complete resection rate in neoadjuvant CRT after a median of 45 -month follow-up. ${ }^{30}$ The updated long-term results published recently continue to show an improved OS in the neoadjuvant CRT group after a median of 84.1-month follow-up, and this improvement was clinically significant for both SCC and AC. ${ }^{36}$

Most studies published previously have also shown a reduction in LRR after preoperative CRT for esophageal cancer compared to surgery alone. ${ }^{26,29,37,38}$ The LRR rates range from $5 \%$ to $14 \%$ and $34 \%$ to $42 \%$ after neoadjuvant CRT and surgery alone, respectively. ${ }^{26,29,37,38}$ The recurrence outcomes in the CROSS study showed that preoperative CRT reduced the overall recurrence rate (surgery $58 \%$ vs preoperative CRT $35 \%$ ) and that preoperative CRT reduced LRR from $34 \%$ to $14 \%(P<0.001)$ and peritoneal carcinomatosis from $14 \%$ to $4 \%(P<0.001)$. Recurrence within the radiation target volume occurred in only $5 \%$, mostly combined with outfield failures. ${ }^{38}$ Neoadjuvant CRT also reduces the risk of distant recurrence. ${ }^{35}$

The meta-analysis of randomized trials comparing neoadjuvant CRT and surgery alone for esophageal cancer also demonstrated that preoperative CRT could improve the survival and local control. ${ }^{39}$ The meta-analysis by Sjoquist et $\mathrm{al}^{40}$ included 12 trials that were randomized comparisons

Table 2 Randomized trials of neoadjuvant CRT vs surgery alone for esophageal cancer

\begin{tabular}{|c|c|c|c|c|c|c|c|c|c|c|c|}
\hline \multirow[t]{2}{*}{ References } & \multicolumn{2}{|l|}{$\mathbf{N}$} & \multirow[t]{2}{*}{ RT dose } & \multirow[t]{2}{*}{ CT } & \multirow[t]{2}{*}{ pCR (\%) } & \multicolumn{3}{|l|}{ DFS (\%) } & \multicolumn{3}{|l|}{ OS (\%) } \\
\hline & CRT & $\mathbf{S}$ & & & & CRT & $\mathbf{s}$ & $P$-value & CRT & $\mathbf{S}$ & $P$-value \\
\hline Tepper et al" & 30 & 26 & $50.4 \mathrm{~Gy} / \mathrm{I} .8 \mathrm{~Gy} / 28 \mathrm{f}$ & $\mathrm{DDP}+5-\mathrm{FU}$ & 33.3 & - & - & - & 5 years: 39 & 5 years: 16 & $<0.05$ \\
\hline Bosset et $\mathrm{al}^{25}$ & 143 & 139 & 37 Gy/3.7 Gy/lof & DPP & 26 & - & - & 0.003 & - & - & 0.78 \\
\hline Urba et $\mathrm{al}^{26}$ & 50 & 50 & $45 \mathrm{~Gy} / \mathrm{l} .5 \mathrm{~Gy} / 30 \mathrm{f}$ & $\begin{array}{l}\mathrm{DDP}+5-\mathrm{FU} \\
+\mathrm{VCR}\end{array}$ & 28 & 3 years: 28 & 3 years: 16 & 0.16 & 3 years: 30 & 3 years: 16 & 0.15 \\
\hline Lee et $\mathrm{al}^{27}$ & 51 & 50 & $45.6 \mathrm{~Gy} / \mathrm{l} .2 \mathrm{~Gy} / 38 \mathrm{f}$ & $\mathrm{DDP}+5-\mathrm{FU}$ & 43 & 2 years: 49 & 2 years: $5 \mathrm{I}$ & 0.93 & 2 years: 55 & 2 years: 57 & 0.69 \\
\hline Burmeister et $\mathrm{a}^{28}$ & 128 & 128 & $35 \mathrm{~Gy} / 2.33 \mathrm{~Gy} / \mathrm{I} 5 \mathrm{f}$ & $\mathrm{DDP}+5-\mathrm{FU}$ & 16 & - & - & - & - & - & 0.57 \\
\hline Lv et $\mathrm{a}^{29}$ & 80 & 80 & $40 \mathrm{~Gy} / 2 \mathrm{~Gy} / 20 \mathrm{f}$ & DDP + PTX & & - & - & - & 5 years: 43.5 & 5 years: 34 & $<0.05$ \\
\hline van Hagen et a $\left.\right|^{30}$ & 178 & 188 & $4 \mathrm{I} .4 \mathrm{~Gy} / \mathrm{I} .8 \mathrm{~Gy} / 23 \mathrm{f}$ & $\mathrm{CBP}+\mathrm{PTX}$ & 29 & 5 years: 44 & 5 years: 27 & $<0.001$ & 5 years: 47 & 5 years: 33 & $<0.05$ \\
\hline Shapiro et $\mathrm{al}^{36}$ & 178 & 188 & $41.4 \mathrm{~Gy} / \mathrm{I} .8 \mathrm{~Gy} / 23 \mathrm{f}$ & $\mathrm{CBP}+\mathrm{PTX}$ & & - & - & - & - & - & 0.003 \\
\hline Apinop et $\mathrm{al}^{31}$ & 35 & 34 & - & $\mathrm{DPP}+5-\mathrm{FU}$ & 27 & - & - & - & - & - & $>0.05$ \\
\hline Le Prise et $\mathrm{al}^{32}$ & 41 & 45 & $20 \mathrm{~Gy} / 2 \mathrm{~Gy} / \mathrm{IOf}$ & $\mathrm{DPP}+5-\mathrm{FU}$ & & - & - & - & - & - & $>0.05$ \\
\hline Walsh et $\mathrm{al}^{33}$ & 58 & 55 & $40 \mathrm{~Gy} / 2.67 \mathrm{~Gy} / \mathrm{I} 5 \mathrm{f}$ & $\mathrm{DPP}+5-\mathrm{FU}$ & 25 & - & - & - & 3 years: 32 & 3 years: 6 & 0.01 \\
\hline Natsugoe et a $\left.\right|^{34}$ & 22 & 23 & $40 \mathrm{~Gy} / 2 \mathrm{~Gy} / 20 \mathrm{f}$ & $\mathrm{DPP}+5-\mathrm{FU}$ & & - & - & - & 5 years: 57 & 5 years: 41 & $>0.05$ \\
\hline Ariga et $\mathrm{a}^{35}$ & 51 & 48 & $60 \mathrm{~Gy} / 2 \mathrm{~Gy} / 30 \mathrm{f}$ & $\mathrm{DPP}+5-\mathrm{FU}$ & & - & - & - & 5 years: 75.7 & 5 years: 51 & 0.0169 \\
\hline
\end{tabular}

Abbreviations: CBP, carboplatin; CRT, chemoradiotherapy; CT, chemotherapy; DDP, cisplatin; DFS, disease-free survival; 5-FU, fluorouracil; OS, overall survival; pCR, pathological complete response rate; PTX, paclitaxel; RT, radiotherapy; S, surgery; VCR, vinblastine. 
of neoadjuvant adjuvant CRT vs surgery alone $(n=1,854)$ in patients with operable esophageal carcinoma and provided strong evidence for a survival benefit of neoadjuvant CRT over surgery alone in patients with SCC and AC of the esophagus.

Neoadjuvant CRT can increase the complete resection rate for esophageal cancer, ${ }^{25,28,30}$ with the rate of preoperative CRT that ranges from $80 \%$ to $92 \%$ compared to the rate of surgery alone that ranges from $59 \%$ to $69 \% .^{28,30}$ The number of positive lymph nodes could also be reduced with preoperative $\mathrm{CRT},{ }^{28}$ which is associated with good survival. ${ }^{41-43}$ Preoperative CRT significantly increases the pathologic complete response ( $\mathrm{pCR}$ ) rate, ranging from $18 \%$ to $43 \%$. $^{27,30,33,44-49} \mathrm{pCR}$ is an independent favorable prognostic factor for survival after surgery ${ }^{40,50-53}$ and is also a favorable prognostic factor for both locoregional and systemic recurrences. ${ }^{40}$

Most studies report that preoperative CRT does not increase postoperative complication and mortality, ${ }^{25,29-32,54}$ and a few studies report that preoperative CRT may increase postoperative complication and mortality. ${ }^{25}$ Bosset et $\mathrm{al}^{25}$ reported that preoperative CRT did not increase postoperative complication but increased postoperative mortality compared to surgery alone (12.3\% vs $4 \%$, respectively); however, the mortality in the preoperative group is very high, which may be associated with surgery procedure. A meta-analysis by Mungo et $\mathrm{al}^{55}$ showed that preoperative therapy for esophageal cancer did not increase 30-day mortality or the overall risk of postoperative complications after esophagectomy. According to these results, preoperative CRT should not be hindered by its postoperative complication and mortality.

According to the evidence discussed earlier, especially with the results of the CROSS trial, preoperative CRT has a long-term survival benefit over surgery alone without increasing postoperative complication and mortality. Thus, neoadjuvant CRT is now considered the standard of care in many parts of the world for the treatment of resectable and locally advanced esophageal cancer, both for SCC and AC.

\section{Neoadjuvant chemotherapy and surgery vs surgery alone}

Preoperative cisplatin-based chemotherapy is an alternative choice for locally advanced esophageal cancer, and it is widely adopted in Europe and parts of the USA, especially in patients with lower esophageal and EGJ cancers. The survival benefit of neoadjuvant chemotherapy in patients with operable esophageal cancer has been reported in a number of trials..$^{456-59}$ The randomized study in the UK demonstrated that preoperative chemotherapy was superior to surgery alone in OS in patients with resectable esophageal cancer without increasing postoperative complications. ${ }^{56}$ The updated longterm results showed maintained survival benefit of preoperative chemotherapy over surgery alone for both AC and SCC of esophagus $(P=0.03$ ) with a 5-year survival of $23.0 \%$ for preoperative chemotherapy compared to $17.1 \%$ for surgery alone. ${ }^{4}$ Some randomized trials suggest that preoperative chemotherapy has a survival benefit in resectable AC of the stomach, EGJ, or lower esophagus; however, esophageal AC only accounted for $10 \%-15 \%$ of the cases. ${ }^{10,59}$ A metaanalysis on preoperative chemotherapy trials in esophageal cancers, including nine randomized comparisons of neoadjuvant chemotherapy vs surgery alone $(n=1,981)$, provided the strongest evidence for a survival benefit of neoadjuvant chemotherapy over surgery alone in patients with esophageal carcinoma with an hazard ratio (HR) of 0.87 (95\% confidence interval [CI], 0.79-0.96; $P=0.005) .{ }^{40}$ Similarly, a Cochrane review including 14 randomized trials of $\mathrm{AC}$ of the stomach, EGJ, and lower esophagus also demonstrated that preoperative chemotherapy was associated with a significantly longer $\mathrm{OS}^{43}$ A meta-analysis reported by Gebski et $\mathrm{al}^{39}$ found that neoadjuvant chemotherapy did not have a survival benefit for patients with SCC (HR for mortality 0.88 [0.75-1.03]; $P=0.12)$, whereas the survival benefit was significant for $\mathrm{AC}$ group (HR for mortality 0.78 [0.64-0.95]; $P=0.014$ ). On the other hand, a US Intergroup trial 0113 revealed no OS benefits for preoperative chemotherapy over surgery alone. ${ }^{41,60}$

Some series reported that preoperative chemotherapy could downstage the tumor and increase the resection rate, which may result in improved survival. ${ }^{4,43,61,62}$ A retrospective study reported that patients with downstaged tumors after neoadjuvant chemotherapy experienced an improved survival compared to patients without response $(P<0.001)$ and the lower rates of local recurrence $(6 \%$ vs $13 \%$, respectively; $P=0.03$ ) and systemic recurrence (19\% vs 29\%, respectively; $P=0.027$ ), and downstaging was the strongest independent predictor of survival. ${ }^{61}$ The updated results of the randomized trial in the UK demonstrated macroscopic residual disease from incomplete resection (R2) or no resection in $26.4 \%$ of surgery vs $14.3 \%$ of the preoperative chemotherapy $(P<0.001) .{ }^{4}$ A review also revealed that preoperative chemotherapy was associated with higher rates of $\mathrm{R} 0$ resection and more favorable tumor stage upon resection. ${ }^{43}$

Some studies have demonstrated that preoperative chemotherapy would not increase serious postoperative complications for esophageal cancer. ${ }^{56,60}$ A review and a meta-analysis also showed that preoperative chemotherapy for esophageal 
carcinoma did not increase the risk of postoperative morbidity or perioperative mortality compared to surgery alone. ${ }^{43,63}$ According to the evidence provided earlier, preoperative chemotherapy is a treatment option for patients with operable esophageal cancer, mainly for AC.

\section{Neoadjuvant CRT vs neoadjuvant chemotherapy for esophageal cancer}

Theoretically, neoadjuvant CRT would be superior to neoadjuvant chemotherapy for esophageal cancer since the addition of radiation to neoadjuvant chemotherapy does increase the pathologic response and complete resection rate, both of which should result in improved treatment outcomes. There is proportionately a larger benefit for neoadjuvant CRT than for neoadjuvant chemotherapy, although both are associated with a survival benefit compared to surgery alone. ${ }^{39}$ The Preoperative Chemotherapy or Radiochemotherapy in Esophago-gastric Adenocarcinoma Trial (POET) was an attempt to compare these two treatment approaches by randomizing patients with locally advanced $\mathrm{AC}$ of the EGJ to either neoadjuvant CRT to $30 \mathrm{~Gy}$ in 15 fractions or neoadjuvant chemotherapy. ${ }^{64}$ Unfortunately, the trial was closed early due to poor accrual after enrolling 126 patients. The 3 -year survival was $47.4 \%$ for preoperative CRT compared to $27.7 \%$ for preoperative chemotherapy, although this was not statistically significant $(P=0.07)$. Nevertheless, compared to preoperative chemotherapy alone, preoperative CRT significantly increased $\mathrm{pCR}$ and the rate of tumor-free lymph nodes ( $15.6 \%$ vs $2.0 \%$ and $64.4 \%$ vs $37.7 \%$, respectively). However, a meta-analysis included only two studies: one of which is POET trial showed weak evidence in favor of neoadjuvant CRT. ${ }^{40}$ Neoadjuvant CRT is accepted as the standard treatment for locally advanced esophageal cancer, both for SCC and AC, but the benefits of neoadjuvant CRT over neoadjuvant chemotherapy for AC of the esophagus remain controversial. More randomized trials are needed to compare the effectiveness of the two modalities.

\section{Neoadjuvant CRT vs definitive CRT for locally advanced esophageal cancer}

Early nonrandomized studies with small sample sizes demonstrated that patients treated with CRT had similar survival rates with or without surgery. ${ }^{65,66}$ To date, there are only two randomized trials that compared definitive CRT with neoadjuvant CRT for locally advanced esophageal cancer. ${ }^{67,68}$ The Fédération Francophone de Cancérologie Digestive (FFCD) 9102 study randomized responding patients after 46 Gy with two cycles of cisplatin/fluorouracil to either surgery or continuation of CRT to additional 20 Gy. The study reported that adding surgery after CRT brought no survival benefit for patients with locally advanced esophageal cancer, although there was a benefit in 2-year local control of $66.4 \%$ compared to $57.0 \%$ for neoadjuvant CRT vs definitive CRT, respectively. ${ }^{68}$ A second multicenter randomized trial in Germany enrolled 172 patients with locally advanced SCC of the esophagus reported that definitive CRT also resulted in equivalent survival, but there was better local progression-free survival in the surgery group $(64.3 \%$ vs $40.7 \% ; P=0.03) .{ }^{67}$ Nevertheless, surgery significantly increased the local tumor control, ${ }^{67,68}$ and patients who underwent surgery had a lower chance of death from cancer. ${ }^{67}$ The benefits of surgery in FFCD 9102 might have been confounded by some methodological issues. ${ }^{69}$ The overall lower dose of chemotherapy for patients receiving resection, the use of split radiation course, which may result in worse survival rates than conventional radiotherapy, ${ }^{70}$ and the high surgical mortality ${ }^{25,71}$ may have negatively impacted OS outcome. Therefore, the benefit of surgery after CRT is still an open question and may need a modern day trial using modern techniques to convincingly answer this question.

\section{The role of salvage surgery after CRT}

Since the question of the need for additional surgery after CRT for locally advanced esophageal cancer is still not convincingly answered, an important question that is raised is which patients can surgery be spared? There are a number of studies that have shown that patients with pCR have better prognosis. ${ }^{40,49-53,67,68,72}$ Berger et al ${ }^{53}$ reported that the 5-year survival of patients who achieved $\mathrm{pCR}$ after preoperative CRT was $\sim 50 \%$. Furthermore, the LRR rate is also low in patients who achieved pCR after neoadjuvant CRT. ${ }^{51,73}$ This suggests that if there is a clear clinical response, which likely enriches for patients with pCR, surgery can probably be avoided at the beginning, and salvage surgery can be done on the patients who have persistent diseases and local or regional recurrence. Salvage surgery after definitive CRT is appropriate and has good outcomes in responding patients. ${ }^{74,75} \mathrm{~A}$ series of data on nonrandomized patients from the FFCD 9102 trial suggest that attempting surgery after early failure of CRT is beneficial and should be considered in patients who are still operable. ${ }^{76}$ A retrospective propensity score-matched study included 65 patients with esophageal AC who underwent salvage esophagectomy after failed definitive CRT and compared this group to 65 matched patients who received planned esophagectomy. The study demonstrated that salvage surgery was feasible for patients with esophageal AC who failed definitive CRT and recurred locoregionally since postopera- 
tive morbidity, mortality, and OS were comparable between the two groups of patients. ${ }^{77}$ Recently, a large multicenter study from the modern era with propensity score matching included 308 patients in definitive CRT plus salvage surgery arm and 540 patients in neoadjuvant CRT plus surgery arm and reported that salvage surgery arm can offer acceptable short- and long-term outcomes in selected patients at experienced centers compared to neoadjuvant CRT plus surgery arm, with 3-year OS (43.3\% vs 40.1\%; $P=0.542$ ), disease-free survival (39.2\% vs 32.8\%; $P=0.232)$, and similar recurrence pattern; the perioperative complication was acceptable. ${ }^{74}$ Therefore, salvage surgery after definitive CRT may be safe and feasible for patients with esophageal cancer who achieve pCR after neoadjuvant CRT at experienced centers.

\section{CRT plus target therapy vs CRT for esophageal cancer}

Epidermal growth factor receptor (EGFR) is overexpressed in $50 \%-80 \%$ of gastric and EGJ $\mathrm{ACs}^{78,79}$ and is associated with poor prognosis. ${ }^{79-81}$ Cetuximab, a monoclonal EGFR antagonist, improved outcomes when given in combination with chemotherapy in some advanced cancers, especially in squamous-cell head and neck cancer. ${ }^{80,81}$ The SCOPE1 trial, a multicenter, randomized, open-label, Phase II/III trial, aimed to investigate the addition of cetuximab to definitive CRT in patients with localized esophageal SCC and ACs, and it was closed when 258 out of 600 patients were enrolled due to interim analysis for futility. The CRT plus cetuximab group had fewer patients with treatment failure free at 24 weeks than the CRT only group (66.4\% and $76.9 \%$, respectively) and also had a shorter median OS (22.1 months vs 25.4 months, respectively; $P=0.035$ ). In addition, the toxicity was worse, and less patients completed therapy with cetuximab. ${ }^{82}$ The addition of cetuximab to definitive CRT cannot be recommended for patients with esophageal cancer.

Human epidermal growth factor receptor 2 (HER2; also known as ERBB2) is an important biomarker and driver of tumorigenesis in gastric cancer, with $7 \%-34 \%$ of overexpression or amplification. ${ }^{83-86}$ Blocking this powerful growth factor signal may improve patient's outcome. The Trastuzumab for Gastric Cancer (ToGA) study is the first multicenter randomized trial to evaluate the efficacy and safety of trastuzumab, a monoclonal antibody that targets HER2, in combination with chemotherapy for the first-line treatment of HER2-positive advanced gastric or gastroesophageal junction cancer $(\sim 80 \%$ of gastric cancer). A total of 594 patients were randomly assigned to study treatment (trastuzumab plus chemotherapy: $\mathrm{n}=298$; chemotherapy alone: $\mathrm{n}=296$ ). Median follow-up was 18.6 months and 17.1 months, respectively. There was a significant improvement in median OS in the trastuzumab plus chemotherapy arm compared to chemotherapy arm alone (13.8 months and 11.1 months, respectively; HR 0.74; 95\% CI $0.60-0.91 ; P=0.0046)$. Toxicity was similar in two arms. This study established trastuzumab in combination with chemotherapy as a new standard option for patients with HER2positive advanced gastric or gastroesophageal junction cancer. OS was longer in patients with high expression (immunohistochemistry $2+$ and fluorescence in situ hybridization [FISH] positive or immunohistochemistry $3+$ ) of HER2 protein than in patients with low expression (immunohistochemistry 0 and FISH positive or immunohistochemistry 1+ and FISH positive) in the post hoc subgroup analysis. ${ }^{87}$ The addition of trastuzumab to chemotherapy substantially improved OS in patients with high expression of HER2 ( $n=446 ; 16$ months vs 11.8 months; $\mathrm{HR}=0.65$ ) compared to those with low expression of HER 2 ( $n=131 ; 10$ months vs 8.7 months; HR $=1.07){ }^{87}$ The addition of trastuzumab to CRT for esophageal cancer is currently being tested in the RTOG 1010 trial to evaluate the effectiveness of trastuzumab in combination with neoadjuvant CRT for esophageal cancer.

\section{Advances in radiation delivery: three- dimensional conformal radiotherapy vs intensity-modulated radiation therapy for esophageal cancer}

Radiotherapy is an extremely effective treatment for esophageal cancer. The advent of three-dimensional conformal radiotherapy (3D-CRT) brought a major shift from conventional, two-dimensional techniques, and it has substituted conventional radiation gradually in clinical practice as the standard treatment for esophageal cancer. However, 3D-CRT is still associated with significant long-term toxicities because of excessive radiation dose to the lungs and heart, which may result in pneumonitis and heart failure and/or cardiac arrhythmia. Intensity-modulated radiation therapy (IMRT) is an advanced radiation technique that uses inverse planning or automated optimization to deliver a nonuniform radiation dose within the radiation field so that the dose could be concentrated within the tumor with relative sparing of the surrounding normal organs. ${ }^{88}$ Therefore, IMRT has significant dosimetric advantages over 3D-CRT.

IMRT has been shown to be superior to 3D-CRT with respect to conformity and dose homogeneity to the target. ${ }^{89-94}$ Most studies reported that IMRT can better spare the lungs with respect to the lung volume receiving $>20 \mathrm{~Gy}$ (V20) $)^{90,91,93,95}$ mean lung dose (MLD), ${ }^{90,91}$ and even V10 and $\mathrm{V} 5 ;{ }^{90,95}$ however, some studies reported that IMRT resulted in larger V10. ${ }^{96}$ Some studies reported that IMRT can reduce the radiation dose to the heart in terms of heart volume exposed 
to $>30 \mathrm{~Gy}$ (V30), ${ }^{93,95-97} 40 \mathrm{~Gy}$ (V40),${ }^{93,95}$ mean heart dose, ${ }^{97}$ and even the right coronary artery. ${ }^{97}$ Thus, IMRT has a much greater potential for achieving a higher degree of target conformity and/or normal tissue sparing than 3D-CRT, especially for target volumes and/or organs at risk with complex shapes and/or concave regions.

Many studies reported that lung V20 and MLD are related to radiation pneumonitis. ${ }^{98-103}$ IMRT can significantly improve lung sparing in terms of the MLD and V20, and theoretically, IMRT could reduce pulmonary complications. Tsujino et al ${ }^{104}$ reported that the 12-month cumulative incidences of radiation pneumonitis greater than grade 2 were $0 \%, 7.1 \%, 25 \%$, and $42.9 \%$ in patients with a V20 of $<20 \%, 21 \%-25 \%, 26 \%-30 \%$, and $>31 \%$, respectively, for 43 patients with small-cell lung cancer receiving CRT. Lee et al demonstrated that radiotherapy techniques, which decrease the volume of lung receiving low radiation doses (V10 and V15), may also significantly reduce the risk of this potentially life-threatening complication.

It is reported that cardiac complications after radiation are related to heart V30, V40 and higher dose volumes, and mean heart dose. ${ }^{105,106}$ Gayed et al ${ }^{106}$ reported a $54 \%$ rate of cardiac ischemia 3 months following CRT for esophageal cancer and found that most perfusion defects were located in the region of the heart receiving $\geq 45 \mathrm{~Gy}$. A study including 101 patients with inoperable esophageal cancer treated with CRT found that the risk of pericardial effusion increased significantly with a mean pericardial dose of $>26.1$ Gy ( $73 \%$ vs $13 \% ; P=0.002)$ and a pericardium V30 of $>46 \%$ ( $73 \%$ vs $13 \% ; P=0.001) .{ }^{105}$ Does the dosimetric advantage of IMRT translate to better clinical outcomes? The largest series was reported by Lin et al ${ }^{107}$ that compared the long-term outcomes with 3D-CRT and IMRT and demonstrated that OS, locoregional control, and cardiac death were significantly better after IMRT than after 3D-CRT. Freilich et al ${ }^{108}$ reported that compared to 3D-CRT, IMRT-based CRT resulted in comparable survival but decreased significantly grade $\geq 3$ toxicity, which is defined as any hospitalization, feeding tube, or $>20 \%$ weight loss (OR $0.51 ; P=0.050$ ).

Although IMRT is now widely adopted for esophageal cancer treatment in clinical practice, there was never a high-quality randomized trial conducted to compare the two modalities. Additional large cohort studies are needed to compare the treatment outcomes of IMRT and 3D-CRT.

\section{Further dosimetric and clinical advantages: proton therapy vs $\mathrm{X}$-ray therapy for esophageal cancer}

The defining physical characteristic of proton depth dose curves is the peak at the end of the proton range where most of the energy from the protons is deposited, beyond which the dose is negligible. This spike in energy deposition is known as the Bragg peak. Through its characteristic Bragg peak, proton therapy has the potential to precisely deliver large and uniform dose to a tumor target while minimizing dose to surrounding healthy tissue, which may decrease the radiation-induced toxicity. The dosimetric advantages of proton therapy for esophageal cancer have been demonstrated in comparison to 3D-CRT and IMRT in several planning studies. ${ }^{109-111}$ As compared to IMRT, both the two-beam and three-beam proton therapies resulted in much better lung sparing, with median lung V5, V10, V20, and MLD reduced by $35.6 \%, 20.5 \%, 5.8 \%$, and 5.1 Gy and $17.4 \%, 8.4 \%, 5 \%$, and $2.9 \mathrm{~Gy}$, respectively. ${ }^{110}$ In a dosimetric comparative study between IMRT and intensity-modulated proton therapy (IMPT), IMPT spared much more of the heart, lung, liver, and spinal cord. ${ }^{111}$ The dosimetric benefits of proton therapy will need to be tailored to each patient according to their specific cardiac and pulmonary risks. In a dosimetric study comparing IMPT with IMRT in esophageal cancer, IMPT produced lower MLD, lung V5 and V20, and heart V40 than did IMRT $(P<0.05) .{ }^{112}$ In another comparative planning study, proton plans showed a decreased dose to various volumes of the heart and lungs, especially lung V5, while achieving adequate target coverage when compared to both the IMRT and 3D-CRT plans. ${ }^{109}$

Furthermore, proton therapy can also improve heart sparing in terms of heart V30 and mean heart dose, which may reduce the risk of radiation-induced cardiovascular toxicity. Ling et a $\mathrm{l}^{109}$ reported that the proton plans delivered a significantly lower dose to the left anterior descending artery and left ventricle in comparison to both the IMRT and 3D-CRT plans. Since irradiation of left anterior descending artery and left ventricle poses a particular risk to develop coronary artery and ischemic heart disease, ${ }^{113}$ proton therapy may also reduce the risk of those diseases. In a 55-patient dosimetric comparison of passive scattering proton plans to IMRT plans for the same patients, proton beam is able to significantly spare the heart, lung, and liver. ${ }^{114}$

The outcomes of proton therapy for esophageal cancer have been reported by many authors. ${ }^{115-121}$ There are some studies which reported that a higher dose of irradiation delivered by proton therapy could result in improved local control and OS. ${ }^{115,120,121}$ In an early Japanese retrospective study, including 15 patients with esophageal cancer treated with proton therapy with or without X-ray therapy, the mean total dose was $80.4 \mathrm{~Gy}$, and the authors found that the high dose of irradiation improved not only the local control but also the long-term survival without increasing the risk of injury to normal organs. ${ }^{120}$ From an update from the same 
institution in 46 patients with esophageal cancer, the median total dose of combined X-ray and proton radiation for 40 patients was $76.0 \mathrm{~Gy}$, and that of the remaining six patients receiving only proton therapy was $82.0 \mathrm{~Gy}$. It reported that the 5-year actuarial survival, disease-specific survival, and local control rates for the 23 patients with $\mathrm{T} 1$ were $55 \%$, $95 \%$, and $83 \%$, respectively, which was much higher than conventional radiotherapy. ${ }^{115}$ The good outcome might be associated with dose escalation, with some patients receiving dose $\geq 80$ Gy. ${ }^{115}$ In another study with 30 patients with esophageal cancer (superficial: 13; locally advanced: 17) treated with proton therapy with or without photon therapy, the mean total doses were 77.7 Gy and 80.7 Gy in the superficial and locally advanced cancers, respectively. ${ }^{121}$ However, all the aforementioned studies are done without chemotherapy. In a prospective study enrolling 62 patients with esophageal cancer who underwent concurrent chemotherapy (CChT) and proton beam therapy (PBT) (CChT/PBT), the preliminary outcomes demonstrated that the response rates to preoperative CChT/PBT were encouraging, with $28 \%$ of the pCR rate for the surgical cohort and $50 \%$ of the pCR and near CR rates ( $0 \%-1 \%$ residual cells). ${ }^{118}$ Another recent single institutional study in 40 patients attested to the similar safety and efficacy of CChT and proton beam therapy. ${ }^{119}$ Because postoperative complications are relatively common after neoadjuvant chemoradiation, particularly pulmonary complications, which relate to the radiation exposure of the heart and lungs, ${ }^{122}$ proton beam therapy could potentially reduce the postoperative toxicities. Indeed, a retrospective study comparing 3D-CRT, IMRT, and proton beam therapy in patients who underwent surgery after neoadjuvant chemoradiation demonstrated significantly improved outcomes in the proton-treated patients compared to 3D-CRT and a strong trend compared to IMRT. ${ }^{123}$

Although proton therapy holds substantial promise to improve the outcomes of esophageal cancer, almost all the studies reported so far are retrospective single-institution series that may limit the reliability of the results. A prospective randomized trial comparing proton therapy and IMRT (NCT01512589) will hopefully provide definitive evidence for the value and benefit of proton therapy.

\section{Conclusion}

Neoadjuvant CRT has been accepted as the standard treatment for esophageal cancer. pCR after CRT is associated with good survival and local control, and surgery may be spared for these patients. However, there are currently no effective mean to predict for $\mathrm{pCR}$, and future efforts will be needed to identify imaging- or blood-based methods to predict for pCR. Although it was a clinical failure to have added EGFR-targeting antibody to standard CRT in an unselected population of patients, there is a lot of hope that the addition of trastuzumab to standard backbone chemotherapy and radiation in HER2+ esophageal AC will improve pCR and clinical outcomes. There is also no high-quality evidence on comparing CRT plus salvage surgery and neoadjuvant CRT plus upfront surgery, and clinical trials may be needed to test this question. IMRT has substituted 3D-CRT as a standard treatment in clinical practice because of its dosimetric benefits. Step-and-shoot IMRT is mainly used, whereas volumetric modulated arc therapy may have some dosimetric and clinical benefits over step-and-shoot IMRT, but the former is currently being explored. Although proton therapy has many dosimetric benefits over photon therapy, but whether the dosimetric benefits could be translated into clinical benefits is still uncertain, and data on radiation therapy of esophageal cancer with protons are still relatively rare. Trials comparing proton therapy to standard photon-based radiation will be needed to answer this question.

\section{Disclosure}

The authors report no conflicts of interest in this work.

\section{References}

1. Ferlay J, Shin HR, Bray F, Forman D, Mathers C, Parkin DM. Estimates of worldwide burden of cancer in 2008: GLOBOCAN 2008. Int J Cancer. 2010;127(12):2893-2917.

2. Siegel R, Naishadham D, Jemal A. Cancer statistics, 2012. CA Cancer J Clin. 2012;62(1):10-29.

3. Berrino F, De Angelis R, Sant M, et al. Survival for eight major cancers and all cancers combined for European adults diagnosed in 1995-99: results of the EUROCARE-4 study. Lancet. 2007;8(9): 773-783.

4. Allum WH, Stenning SP, Bancewicz J, Clark PI, Langley RE. Long-term results of a randomized trial of surgery with or without preoperative chemotherapy in esophageal cancer. J Clin Oncol. 2009;27(30):5062-5067.

5. Kato H, Tachimori Y, Watanabe H, Igaki H, Nakanishi Y, Ochiai A. Recurrent esophageal carcinoma after esophagectomy with three-field lymph node dissection. J Surg Oncol. 1996;61(4):267-272.

6. Nakagawa S, Kanda T, Kosugi S, Ohashi M, Suzuki T, Hatakeyama K. Recurrence pattern of squamous cell carcinoma of the thoracic esophagus after extended radical esophagectomy with three-field lymphadenectomy. J Am Coll Surg. 2004;198(2):205-211.

7. Baba M, Aikou T, Yoshinaka H, et al. Long-term results of subtotal esophagectomy with three-field lymphadenectomy for carcinoma of the thoracic esophagus. Ann Surg. 1994;219(3):310-316.

8. Bhansali MS, Fujita H, Kakegawa T, et al. Pattern of recurrence after extended radical esophagectomy with three-field lymph node dissection for squamous cell carcinoma in the thoracic esophagus. World J Surg. 1997;21(3):275-281

9. Dubecz A, Gall I, Solymosi N, et al. Temporal trends in long-term survival and cure rates in esophageal cancer: a SEER database analysis. JThorac Oncol. 2012;7(2):443-447. 
10. Cen P, Banki F, Cheng L, et al. Changes in age, stage distribution, and survival of patients with esophageal adenocarcinoma over three decades in the United States. Ann Surg Oncol. 2012;19(5):1685-1691.

11. Tepper J, Krasna MJ, Niedzwiecki D, et al. Phase III trial of trimodality therapy with cisplatin, fluorouracil, radiotherapy, and surgery compared with surgery alone for esophageal cancer: CALGB 9781. J Clin Oncol. 2008;26(7):1086-1092.

12. Cooper JS, Guo MD, Herskovic A, et al. Chemoradiotherapy of locally advanced esophageal cancer: long-term follow-up of a prospective randomized trial (RTOG 85-01). Radiation Therapy Oncology Group. JAMA. 1999;281(17):1623-1627.

13. Al-Sarraf M, Martz K, Herskovic A, et al. Progress report of combined chemoradiotherapy versus radiotherapy alone in patients with esophageal cancer: an intergroup study. J Clin Oncol. 1997;15(1):277-284.

14. Herskovic A, Martz K, Al-Sarraf M, et al. Combined chemotherapy and radiotherapy compared with radiotherapy alone in patients with cancer of the esophagus. N Engl J Med. 1992;326(24):1593-1598.

15. Smith TJ, Ryan LM, Douglass HO Jr, et al. Combined chemoradiotherapy vs. radiotherapy alone for early stage squamous cell carcinoma of the esophagus: a study of the Eastern Cooperative Oncology Group. Int J Radiat Oncol Biol Phys. 1998;42(2):269-276.

16. Smit JK, Muijs CT, Burgerhof JG, et al. Survival after definitive (chemo) radiotherapy in esophageal cancer patients: a population-based study in the north-East Netherlands. Ann Surg Oncol. 2013;20(6):1985-1992.

17. Zhu LL, Yuan L, Wang H, et al. A meta-analysis of concurrent chemoradiotherapy for advanced esophageal cancer. PLoS One. 2015;10(6):e0128616.

18. Chen MF, Yang YH, Lai CH, Chen PC, Chen WC. Outcome of patients with esophageal cancer: a nationwide analysis. Ann Surg Oncol. 2013;20(9):3023-3030.

19. Badwe RA, Sharma V, Bhansali MS, et al. The quality of swallowing for patients with operable esophageal carcinoma: a randomized trial comparing surgery with radiotherapy. Cancer. 1999;85(4):763-768.

20. Higuchi K, Komori S, Tanabe S, et al; Kitasato Digestive Disease and Oncology Group. Definitive chemoradiation therapy with docetaxel, cisplatin, and 5-fluorouracil (DCF-R) in advanced esophageal cancer: a phase 2 trial (KDOG 0501-P2). Int J Radiat Oncol Biol Phys. 2014; 89(4):872-879.

21. Chan A, Wong A. Is combined chemotherapy and radiation therapy equally effective as surgical resection in localized esophageal carcinoma? Int J Radiat Oncol Biol Phys. 1999;45(2):265-270.

22. Karran A, Blake P, Chan D, et al. Propensity score analysis of oesophageal cancer treatment with surgery or definitive chemoradiotherapy. Br J Surg. 2014;101(5):502-510.

23. Teoh AY, Chiu PW, Yeung WK, Liu SY, Wong SK, Ng EK. Long-term survival outcomes after definitive chemoradiation versus surgery in patients with resectable squamous carcinoma of the esophagus: results from a randomized controlled trial. Ann Oncol. 2013;24(1):165-171.

24. Pottgen C, Stuschke M. Radiotherapy versus surgery within multimodality protocols for esophageal cancer - a meta-analysis of the randomized trials. Cancer Treat Rev. 2012;38(6):599-604.

25. Bosset JF, Gignoux M, Triboulet JP, et al. Chemoradiotherapy followed by surgery compared with surgery alone in squamous-cell cancer of the esophagus. N Engl J Med. 1997;337(3):161-167.

26. Urba SG, Orringer MB, Turrisi A, Iannettoni M, Forastiere A, Strawderman M. Randomized trial of preoperative chemoradiation versus surgery alone in patients with locoregional esophageal carcinoma. J Clin Oncol. 2001;19(2):305-313.

27. Lee JL, Park SI, Kim SB, et al. A single institutional phase III trial of preoperative chemotherapy with hyperfractionation radiotherapy plus surgery versus surgery alone for resectable esophageal squamous cell carcinoma. Ann Oncol. 2004;15(6):947-954.

28. Burmeister BH, Smithers BM, Gebski V, et al; Trans-Tasman Radiation Oncology Group; Australasian Gastro-Intestinal Trials Group. Surgery alone versus chemoradiotherapy followed by surgery for resectable cancer of the oesophagus: a randomised controlled phase III trial. Lancet Oncol. 2005;6(9):659-668.
29. Lv J, Cao XF, Zhu B, Ji L, Tao L, Wang DD. Long-term efficacy of perioperative chemoradiotherapy on esophageal squamous cell carcinoma. World J Gastroenterol. 2010;16(13):1649-1654.

30. van Hagen P, Hulshof MC, van Lanschot JJ, et al; CROSS Group. Preoperative chemoradiotherapy for esophageal or junctional cancer. N Engl J Med. 2012;366(22):2074-2084.

31. Apinop C, Puttisak P, Preecha N. A prospective study of combined therapy in esophageal cancer. Hepatogastroenterology. 1994;41(4): 391-393.

32. Le Prise E, Etienne PL, Meunier B, et al. A randomized study of chemotherapy, radiation therapy, and surgery versus surgery for localized squamous cell carcinoma of the esophagus. Cancer. 1994; 73(7):1779-1784.

33. Walsh TN, Noonan N, Hollywood D, Kelly A, Keeling N, Hennessy TP. A comparison of multimodal therapy and surgery for esophageal adenocarcinoma. N Engl J Med. 1996;335(7):462-467.

34. Natsugoe S, Okumura H, Matsumoto M, et al. Randomized controlled study on preoperative chemoradiotherapy followed by surgery versus surgery alone for esophageal squamous cell cancer in a single institution. Dis Esophagus. 2006;19(6):468-472.

35. Ariga H, Nemoto K, Miyazaki S, et al. Prospective comparison of surgery alone and chemoradiotherapy with selective surgery in resectable squamous cell carcinoma of the esophagus. Int J Radiat Oncol Biol Phys. 2009;75(2):348-356.

36. Shapiro J, van Lanschot JJ, Hulshof MC, et al. Neoadjuvant chemoradiotherapy plus surgery versus surgery alone for oesophageal or junctional cancer (CROSS): long-term results of a randomised controlled trial. Lancet Oncol. 2015;16(9):1090-1098.

37. Sudo K, Taketa T, Correa AM, et al. Locoregional failure rate after preoperative chemoradiation of esophageal adenocarcinoma and the outcomes of salvage strategies. J Clin Oncol. 2013;31(34): 4306-4310.

38. Oppedijk V, van der Gaast A, van Lanschot JJ, et al. Patterns of recurrence after surgery alone versus preoperative chemoradiotherapy and surgery in the CROSS trials. J Clin Oncol. 2014;32(5):385-391.

39. Gebski V, Burmeister B, Smithers BM, et al; Australasian Gastro-Intestinal Trials Group. Survival benefits from neoadjuvant chemoradiotherapy or chemotherapy in oesophageal carcinoma: a meta-analysis. Lancet. 2007;8(3):226-234.

40. Sjoquist KM, Burmeister BH, Smithers BM, et al; Australasian GastroIntestinal Trials Group. Survival after neoadjuvant chemotherapy or chemoradiotherapy for resectable oesophageal carcinoma: an updated meta-analysis. Lancet. 2011;12(7):681-692.

41. Kelsen DP, Winter KA, Gunderson LL, et al; Radiation Therapy Oncology Group; USA Intergroup. Long-term results of RTOG trial 8911 (USA Intergroup 113): a random assignment trial comparison of chemotherapy followed by surgery compared with surgery alone for esophageal cancer. J Clin Oncol. 2007;25(24):3719-3725.

42. Hamilton E, Vohra RS, Griffiths EA. What is the best neoadjuvant regimen prior to oesophagectomy: chemotherapy or chemoradiotherapy? Int J Surg. 2014;12(3):196-199.

43. Ronellenfitsch U, Schwarzbach M, Hofheinz R, et al. Perioperative chemo(radio)therapy versus primary surgery for resectable adenocarcinoma of the stomach, gastroesophageal junction, and lower esophagus. Cochrane Database Syst Rev. 2013;5:CD008107.

44. Luc G, Gronnier C, Lebreton G, et al. Predictive factors of recurrence in patients with pathological complete response after esophagectomy following neoadjuvant chemoradiotherapy for esophageal cancer: a multicenter study. Ann Surg Oncol. 2015;22(suppl 3):1357-1364.

45. Meluch AA, Greco FA, Gray JR, et al. Preoperative therapy with concurrent paclitaxel/carboplatin/infusional 5-FU and radiation therapy in locoregional esophageal cancer: final results of a Minnie Pearl Cancer Research Network phase II trial. Cancer J. 2003;9(4):251-260.

46. Mandard AM, Dalibard F, Mandard JC, et al. Pathologic assessment of tumor regression after preoperative chemoradiotherapy of esophageal carcinoma. Clinicopathologic correlations. Cancer. 1994;73(11):2680-2686. 
47. Darnton SJ, Archer VR, Stocken DD, Mulholland PJ, Casson AG, Ferry DR. Preoperative mitomycin, ifosfamide, and cisplatin followed by esophagectomy in squamous cell carcinoma of the esophagus: pathologic complete response induced by chemotherapy leads to long-term survival. J Clin Oncol. 2003;21(21):4009-4015.

48. Meredith KL, Weber JM, Turaga KK, et al. Pathologic response after neoadjuvant therapy is the major determinant of survival in patients with esophageal cancer. Ann Surg Oncol. 2010;17(4):1159-1167.

49. Zanoni A, Verlato G, Giacopuzzi S, et al. Neoadjuvant concurrent chemoradiotherapy for locally advanced esophageal cancer in a single high-volume center. Ann Surg Oncol. 2013;20(6):1993-1999.

50. Morita M, Masuda T, Okada S, et al. Preoperative chemoradiotherapy for esophageal cancer: factors associated with clinical response and postoperative complications. Anticancer Res. 2009;29(7):2555-2562.

51. Rohatgi PR, Swisher SG, Correa AM, et al. Failure patterns correlate with the proportion of residual carcinoma after preoperative chemoradiotherapy for carcinoma of the esophagus. Cancer. 2005; 104(7):1349-1355.

52. Swisher SG, Hofstetter W, Wu TT, et al. Proposed revision of the esophageal cancer staging system to accommodate pathologic response (pP) following preoperative chemoradiation (CRT). Ann Surg. 2005;241(5):810-817; discussion 817-820.

53. Berger AC, Farma J, Scott WJ, et al. Complete response to neoadjuvant chemoradiotherapy in esophageal carcinoma is associated with significantly improved survival. J Clin Oncol. 2005;23(19):4330-4337.

54. Saeki H, Morita M, Nakashima Y, et al. Neoadjuvant chemoradiotherapy for clinical stage II-III esophageal squamous cell carcinoma. Anticancer Res. 2011;31(9):3073-3077.

55. Mungo B, Molena D, Stem M, et al. Does neoadjuvant therapy for esophageal cancer increase postoperative morbidity or mortality? Dis Esophagus. 2015;28(7):644-651.

56. Medical Research Council Oesophageal Cancer Working Group. Surgical resection with or without preoperative chemotherapy in oesophageal cancer: a randomised controlled trial. Lancet. 2002;359(9319):1727-1733.

57. Boonstra JJ, Kok TC, Wijnhoven BP, et al. Chemotherapy followed by surgery versus surgery alone in patients with resectable oesophageal squamous cell carcinoma: long-term results of a randomized controlled trial. BMC Cancer. 2011;11:181.

58. Ychou M, Boige V, Pignon JP, et al. Perioperative chemotherapy compared with surgery alone for resectable gastroesophageal adenocarcinoma: an FNCLCC and FFCD multicenter phase III trial. J Clin Oncol. 2011;29(13):1715-1721.

59. Cunningham D, Allum WH, Stenning SP, et al; MAGIC Trial Participants. Perioperative chemotherapy versus surgery alone for resectable gastroesophageal cancer. $N$ Engl J Med. 2006;355(1):11-20.

60. Kelsen DP, Ginsberg R, Pajak TF, et al. Chemotherapy followed by surgery compared with surgery alone for localized esophageal cancer. N Engl J Med. 1998;339(27):1979-1984.

61. Davies AR, Gossage JA, Zylstra J, et al. Tumor stage after neoadjuvant chemotherapy determines survival after surgery for adenocarcinoma of the esophagus and esophagogastric junction. J Clin Oncol. 2014;32(27):2983-2990.

62. Carey RW, Hilgenberg AD, Wilkins EW Jr, et al. Long-term follow-up of neoadjuvant chemotherapy with 5-fluorouracil and cisplatin with surgical resection and possible postoperative radiotherapy and/or chemotherapy in squamous cell carcinoma of the esophagus. Cancer Invest. 1993;11(2):99-105.

63. Kumagai K, Rouvelas I, Tsai JA, et al. Meta-analysis of postoperative morbidity and perioperative mortality in patients receiving neoadjuvant chemotherapy or chemoradiotherapy for resectable oesophageal and gastro-oesophageal junctional cancers. Br J Surg. 2014;101(4): 321-338.

64. Stahl M, Walz MK, Stuschke M, et al. Phase III comparison of preoperative chemotherapy compared with chemoradiotherapy in patients with locally advanced adenocarcinoma of the esophagogastric junction. J Clin Oncol. 2009;27(6):851-856.
65. Algan O, Coia LR, Keller SM, et al. Management of adenocarcinoma of the esophagus with chemoradiation alone or chemoradiation followed by esophagectomy: results of sequential nonrandomized phase II studies. Int J Radiat Oncol Biol Phys. 1995;32(3):753-761.

66. Denham JW, Burmeister BH, Lamb DS, et al. Factors influencing outcome following radio-chemotherapy for oesophageal cancer. The Trans Tasman Radiation Oncology Group (TROG). Radiother Oncol. 1996;40(1):31-43.

67. Stahl M, Stuschke M, Lehmann N, et al. Chemoradiation with and without surgery in patients with locally advanced squamous cell carcinoma of the esophagus. J Clin Oncol. 2005;23(10):2310-2317.

68. Bedenne L, Michel P, Bouche O, et al. Chemoradiation followed by surgery compared with chemoradiation alone in squamous cancer of the esophagus: FFCD 9102. J Clin Oncol. 2007;25(10): $1160-1168$.

69. Rades D, Schild SE. Is surgery necessary following chemoradiation for patients with locally advanced cancer of the esophagus? Nat Clin Pract Oncol. 2007;4(9):506-507.

70. Wobbes T, Baron B, Paillot B, et al. Prospective randomised study of split-course radiotherapy versus cisplatin plus split-course radiotherapy in inoperable squamous cell carcinoma of the oesophagus. Eur J Cancer. 2001;37(4):470-477.

71. Jacob J, Seitz J, Langlois C, Raoul J, Bardet E, Bouché O. Definitive concurrent chemoradiation therapy in squamous cell oesophageal cancer. Results of a french randomized trial comparing standard versus split-course irradiation (FNCLCC-FFCD 9305). Proc Am Soc Clin Oncol. 1999;18:270a.

72. Verlato G, Zanoni A, Tomezzoli A, et al. Response to induction therapy in oesophageal and cardia carcinoma using Mandard tumour regression grade or size of residual foci. Br J Surg. 2010;97(5):719-725.

73. Rohatgi P, Swisher SG, Correa AM, et al. Characterization of pathologic complete response after preoperative chemoradiotherapy in carcinoma of the esophagus and outcome after pathologic complete response. Cancer. 2005;104(11):2365-2372.

74. Markar S, Gronnier C, Duhamel A, et al. Salvage surgery after chemoradiotherapy in the management of esophageal cancer: is it a viable therapeutic option? J Clin Oncol. 2015;33(33):3866-3873.

75. Sudo K, Xiao L, Wadhwa R, et al. Importance of surveillance and success of salvage strategies after definitive chemoradiation in patients with esophageal cancer. J Clin Oncol. 2014;32(30):3400-3405.

76. Vincent J, Mariette C, Pezet D, et al. Early surgery for failure after chemoradiation in operable thoracic oesophageal cancer. Analysis of the non-randomised patients in FFCD 9102 phase III trial: chemoradiation followed by surgery versus chemoradiation alone. Eur J Cancer. 2015;51(13):1683-1693.

77. Marks JL, Hofstetter W, Correa AM, et al. Salvage esophagectomy after failed definitive chemoradiation for esophageal adenocarcinoma. Ann Thorac Surg. 2012;94(4):1126-1132; discussion 1132-1123.

78. Dragovich T, McCoy S, Fenoglio-Preiser CM, et al. Phase II trial of erlotinib in gastroesophageal junction and gastric adenocarcinomas: SWOG 0127. Journal of clinical oncology: official journal of the American Society of Clinical Oncology. October 20, 2006;24(30): 4922-4927.

79. Galizia G, Lieto E, Orditura M, et al. Epidermal growth factor receptor (EGFR) expression is associated with a worse prognosis in gastric cancer patients undergoing curative surgery. World journal of surgery. Jul 2007;31(7):1458-1468.

80. Vermorken JB, Mesia R, Rivera F, et al. Platinum-based chemotherapy plus cetuximab in head and neck cancer. The New England journal of medicine. September 11, 2008;359(11):1116-1127.

81. Bonner JA, Harari PM, Giralt J, et al. Radiotherapy plus cetuximab for squamous-cell carcinoma of the head and neck. The New England journal of medicine. February 9, 2006;354(6):567-578.

82. Crosby T, Hurt CN, Falk S, et al. Chemoradiotherapy with or without cetuximab in patients with oesophageal cancer (SCOPE1): a multicentre, phase 2/3 randomised trial. The Lancet. Oncology. Jun 2013;14(7):627-637. 
83. Gravalos C, Jimeno A. HER2 in gastric cancer: a new prognostic factor and a novel therapeutic target. Annals of oncology: official journal of the European Society for Medical Oncology / ESMO. Sep 2008; 19(9):1523-1529.

84. Hofmann M, Stoss O, Shi D, et al. Assessment of a HER2 scoring system for gastric cancer: results from a validation study. Histopathology. Jun 2008;52(7):797-805.

85. Tanner M, Hollmen M, Junttila TT, et al. Amplification of HER-2 in gastric carcinoma: association with Topoisomerase IIalpha gene amplification, intestinal type, poor prognosis and sensitivity to trastuzumab. Annals of oncology: official journal of the European Society for Medical Oncology / ESMO. Feb 2005;16(2):273-278.

86. Yano T, Doi T, Ohtsu A, et al. Comparison of HER2 gene amplification assessed by fluorescence in situ hybridization and HER2 protein expression assessed by immunohistochemistry in gastric cancer. Oncology reports. Jan 2006;15(1):65-71.

87. Bang YJ, Van Cutsem E, Feyereislova A, et al. Trastuzumab in combination with chemotherapy versus chemotherapy alone for treatment of HER2-positive advanced gastric or gastro-oesophageal junction cancer (ToGA): a phase 3, open-label, randomised controlled trial. Lancet. August 28, 2010;376(9742):687-697.

88. Wu Q, Manning M, Schmidt-Ullrich R, Mohan R. The potential for sparing of parotids and escalation of biologically effective dose with intensity-modulated radiation treatments of head and neck cancers: a treatment design study. Int J Radiat Oncol Biol Phys. 2000;46(1):195-205.

89. Fenkell L, Kaminsky I, Breen S, Huang S, Van Prooijen M, Ringash J. Dosimetric comparison of IMRT vs. 3D conformal radiotherapy in the treatment of cancer of the cervical esophagus. Radiother Oncol. 2008;89(3):287-291.

90. Chandra A, Guerrero TM, Liu HH, et al. Feasibility of using intensitymodulated radiotherapy to improve lung sparing in treatment planning for distal esophageal cancer. Radiother Oncol. 2005;77(3):247-253.

91. Nicolini G, Ghosh-Laskar S, Shrivastava SK, et al. Volumetric modulation arc radiotherapy with flattening filter-free beams compared with static gantry IMRT and 3D conformal radiotherapy for advanced esophageal cancer: a feasibility study. Int J Radiat Oncol Biol Phys. 2012;84(2):553-560.

92. Wu VW, Kwong DL, Sham JS. Target dose conformity in 3-dimensional conformal radiotherapy and intensity modulated radiotherapy. Radiother Oncol. 2004;71(2):201-206.

93. Wang D, Yang Y, Zhu J, Li B, Chen J, Yin Y. 3D-conformal RT, fixedfield IMRT and RapidArc, which one is better for esophageal carcinoma treated with elective nodal irradiation. Technol Cancer Res Treat. 2011;10(5):487-494.

94. Vivekanandan N, Sriram P, Kumar SA, Bhuvaneswari N, Saranya K. Volumetric modulated arc radiotherapy for esophageal cancer. Med Dosim. 2012;37(1):108-113.

95. Gallardo N, Maneru F, Fuentemilla N, et al. SU-E-T-25: a dosimetric comparison of three-dimension conformal and intensity-modulated radiation therapy in esophageal cancer. Med Phys. 2015;42(6):3336.

96. Chen YJ, Liu A, Han C, et al. Helical tomotherapy for radiotherapy in esophageal cancer: a preferred plan with better conformal target coverage and more homogeneous dose distribution. Med Dosim. 2007;32(3):166-171.

97. Kole TP, Aghayere O, Kwah J, Yorke ED, Goodman KA. Comparison of heart and coronary artery doses associated with intensitymodulated radiotherapy versus three-dimensional conformal radiotherapy for distal esophageal cancer. Int J Radiat Oncol Biol Phys. 2012;83(5):1580-1586.

98. Graham MV, Purdy JA, Emami B, et al. Clinical dose-volume histogram analysis for pneumonitis after 3D treatment for non-small cell lung cancer (NSCLC). Int J Radiat Oncol Biol Phys. 1999;45(2): 323-329.

99. Hernando ML, Marks LB, Bentel GC, et al. Radiation-induced pulmonary toxicity: a dose-volume histogram analysis in 201 patients with lung cancer. Int J Radiat Oncol Biol Phys. 2001;51(3):650-659.
100. Kwa SL, Lebesque JV, Theuws JC, et al. Radiation pneumonitis as a function of mean lung dose: an analysis of pooled data of 540 patients. Int J Radiat Oncol Biol Phys. 1998;42(1):1-9.

101. Seppenwoolde Y, Lebesque JV, de Jaeger K, et al. Comparing different NTCP models that predict the incidence of radiation pneumonitis. Normal tissue complication probability. Int J Radiat Oncol Biol Phys. 2003;55(3):724-735.

102. Semenenko VA, Molthen RC, Li C, et al. Irradiation of varying volumes of rat lung to same mean lung dose: a little to a lot or a lot to a little? Int J Radiat Oncol Biol Phys. 2008;71(3):838-847.

103. Zhao L, Ji W, Ou G, et al. Risk factors for radiation-induced lung toxicity in patients with non-small cell lung cancer who received postoperative radiation therapy. Lung Cancer. 2012;77(2):326-330.

104. Tsujino K, Hirota S, Kotani Y, et al. Radiation pneumonitis following concurrent accelerated hyperfractionated radiotherapy and chemotherapy for limited-stage small-cell lung cancer: dose-volume histogram analysis and comparison with conventional chemoradiation. Int J Radiat Oncol Biol Phys. 2006;64(4):1100-1105.

105. Wei X, Liu HH, Tucker SL, et al. Risk factors for pericardial effusion in inoperable esophageal cancer patients treated with definitive chemoradiation therapy. Int J Radiat Oncol Biol Phys. 2008;70(3):707-714.

106. Gayed IW, Liu HH, Yusuf SW, et al. The prevalence of myocardial ischemia after concurrent chemoradiation therapy as detected by gated myocardial perfusion imaging in patients with esophageal cancer. J Nucl Med. 2006;47(11):1756-1762.

107. Lin SH, Wang L, Myles B, et al. Propensity score-based comparison of long-term outcomes with 3-dimensional conformal radiotherapy vs intensity-modulated radiotherapy for esophageal cancer. Int J Radiat Oncol Biol Phys. 2012;84(5):1078-1085.

108. Freilich J, Hoffe SE, Almhanna K, et al. Comparative outcomes for three-dimensional conformal versus intensity-modulated radiation therapy for esophageal cancer. Dis Esophagus. 2015;28(4): $352-357$.

109. Ling TC, Slater JM, Nookala P, et al. Analysis of intensity-modulated radiation therapy (IMRT), proton and 3D conformal radiotherapy (3D-CRT) for reducing perioperative cardiopulmonary complications in esophageal cancer patients. Cancers (Basel). 2014;6(4): 2356-2368.

110. Zhang X, Zhao KL, Guerrero TM, et al. Four-dimensional computed tomography-based treatment planning for intensity-modulated radiation therapy and proton therapy for distal esophageal cancer. Int $J$ Radiat Oncol Biol Phys. 2008;72(1):278-287.

111. Welsh J, Gomez D, Palmer MB, et al. Intensity-modulated proton therapy further reduces normal tissue exposure during definitive therapy for locally advanced distal esophageal tumors: a dosimetric study. Int J Radiat Oncol Biol Phys. 2011;81(5):1336-1342.

112. Chang JY, Li H, Zhu XR, et al. Clinical implementation of intensity modulated proton therapy for thoracic malignancies. Int J Radiat Oncol Biol Phys. 2014;90(4):809-818.

113. Darby SC, Cutter DJ, Boerma M, et al. Radiation-related heart disease: current knowledge and future prospects. Int J Radiat Oncol Biol Phys. 2010;76(3):656-665.

114. Wang J, Palmer M, Bilton SD, et al. Comparing proton beam to intensity modulated radiation therapy planning in esophageal cancer. Int $J$ Particle Ther. 2015;1(4):866-877.

115. Sugahara S, Tokuuye K, Okumura T, et al. Clinical results of proton beam therapy for cancer of the esophagus. Int J Radiat Oncol Biol Phys. 2005;61(1):76-84.

116. Mizumoto M, Sugahara S, Nakayama H, et al. Clinical results of proton-beam therapy for locoregionally advanced esophageal cancer. Strahlenther Onkol. 2010;186(9):482-488.

117. Mizumoto M, Sugahara S, Okumura T, et al. Hyperfractionated concomitant boost proton beam therapy for esophageal carcinoma. Int $J$ Radiat Oncol Biol Phys. 2011;81(4):e601-e606.

118. Lin SH, Komaki R, Liao Z, et al. Proton beam therapy and concurrent chemotherapy for esophageal cancer. Int J Radiat Oncol Biol Phys. 2012;83(3):e345-e351. 
119. Ishikawa H, Hashimoto T, Moriwaki T, et al. Proton beam therapy combined with concurrent chemotherapy for esophageal cancer. Anticancer Res. 2015;35(3):1757-1762.

120. Koyama S, Tsujii H, Yokota H, et al. Proton beam therapy for patients with esophageal carcinoma. Jpn J Clin Oncol. 1994;24(3):144-153.

121. Koyama S, Tsujii H. Proton beam therapy with high-dose irradiation for superficial and advanced esophageal carcinomas. Clin Cancer Res. 2003;9(10 Pt 1):3571-3577.
122. Wang SL, Liao Z, Vaporciyan AA, et al. Investigation of clinical and dosimetric factors associated with postoperative pulmonary complications in esophageal cancer patients treated with concurrent chemoradiotherapy followed by surgery. Int J Rad Oncol Biol Phys. 2006;64(3):692-699.

123. Wang J, Wei C, Tucker SL, et al. Predictors of postoperative complications after trimodality therapy for esophageal cancer. Int J Radiat Oncol Biol Phys. 2013;86(5):885-891.

\section{Publish your work in this journal}

Comparative Effectiveness Research is an international, peer reviewed open access journal focusing on comparative effectiveness of health care including preventative health care strategies, diagnostic strategies, diagnostic technology, medical devices, drugs, medical technology, health systems and organization. The manuscript management system is completely online and includes a very quick and fair peer-review system. Visit http://www.dovepress.com/testimonials.php to read real quotes from published authors.

\footnotetext{
Submit your manuscript here: http://www.dovepress.com/comparative-effectiveness-research-journal
} 